\title{
Dynamics and dietary risk assessment of thiamethoxam in wheat, lettuce and tomato using field experiments and computational simulation
}

\author{
Pang, Nannan; Fan, Xueqi; Fantke, Peter; Zhao, Shengming; Hu, Jiye
}

Published in:

Environmental Pollution

Link to article, DOI:

10.1016/j.envpol.2019.113285

Publication date:

2020

Document Version

Peer reviewed version

Link back to DTU Orbit

Citation (APA):

Pang, N., Fan, X., Fantke, P., Zhao, S., \& Hu, J. (2020). Dynamics and dietary risk assessment of thiamethoxam in wheat, lettuce and tomato using field experiments and computational simulation. Environmental Pollution, 256, [113285]. https://doi.org/10.1016/j.envpol.2019.113285

\section{General rights}

Copyright and moral rights for the publications made accessible in the public portal are retained by the authors and/or other copyright owners and it is a condition of accessing publications that users recognise and abide by the legal requirements associated with these rights.

- Users may download and print one copy of any publication from the public portal for the purpose of private study or research.

- You may not further distribute the material or use it for any profit-making activity or commercial gain

- You may freely distribute the URL identifying the publication in the public portal 


\title{
Dynamics and dietary risk assessment of thiamethoxam
}

\section{in wheat, lettuce and tomato using field experiments and}

\section{computational simulation}

\author{
Nannan Pang ${ }^{1}$, Xueqi Fan ${ }^{1}$, Peter Fantke ${ }^{2}$, Shengming Zhao ${ }^{1}$, and Jiye $\mathrm{Hu}^{1, *}$ \\ ${ }^{1}$ Lab of Pesticide Residues and Environmental Toxicology, School of Chemistry and Biological \\ Engineering, University of Science and Technology Beijing, Beijing 100083, P. R.China \\ ${ }^{2}$ Quantitative Sustainability Assessment, Department of Technology, Management and Economics, \\ Technical University of Denmark, Produktionstorvet 424, 2800 Kgs. Lyngby, Denmark
}

*CORRESPONDING AUTHOR. Professor Jiye Hu (jyhu@ustb.edu.cn), Lab of Pesticide Residues and Environmental Toxicology, School of Chemistry and Biological Engineering, University of Science and Technology Beijing, Beijing 100083, P. R. China, E-mail: jyhu@,ustb.edu.cn. Tel:+86-1082376002 Fax:+86-10-82376002

E-mail addresses:sevensmiling@gmail.com (N. Pang), fanxueqi333@163.com (X. Fan), pefan@dtu.dk (P. Fantke), ustbzsm@163.com (S.Zhao), jyhu@ustb.edu.cn (J. Hu)

\section{Declarations of interest: none}


2 Abstract

3 Thiamethoxam is a widely used pesticide applied to different field crops. To inform risk assessment for

4 this pesticide across relevant crops, we usually rely on field trials, which require time, cost and energy.

5 For providing reliable data across crops and reduce experimental efforts, field trials should be

6 complemented with dynamic modeling. In the present work, we hence focused on combining field trials

7 with dynamic modeling to simulate mass evolutions of the pesticide-plant-system for thiamethoxam

8 applied to wheat, lettuce and tomato as three major food crops. Field trials were conducted with

9 QuEChERS (quick, easy, cheap, effective, rugged and safe) liquid chromatography-mass spectrometry,

10 which gave consistent maximum residue concentrations for thiamethoxam in wheat, lettuce and tomato.

11 We used these residues to evaluate the related dietary risk of humans consuming these food crops. Our

12 results indicated that thiamethoxam did not provide any unacceptable dietary risk for humans across

13 these three food crops, which was in line with other studies. Results for the studied crops could be

14 extrapolated to other crops and with that, our study constituted a cost- and time-efficient way of

15 providing reliable input for risk assessment of pesticides across crops, which was relevant for both

16 practitioners and regulators.

17 Keywords: dietary risk assessment; dynamiCROP; liquid chromatography mass spectrometry; pesticide

18 residue; thiamethoxam 
Pesticides are widely used to increase crop yields. However, it is also well known that pesticide residues put burden on not only the environment but also on humans by pathways including wind, rainfall, surface runoff, and especially food intake of treated cereals, fruits, vegetables, and other food crops (Fantke, 2012a; Fantke and Jolliet, 2016). Thus, pesticide residue analysis and dietary risk assessment have been a global focus for the management and control of not only environmental sample preparation and complex confirmatory techniques such as mass spectrometry (MS) (Hajslova et al., 2011). Relying exclusively on field trials that are usually a representation of specific local soil, weather and crop growth conditions at a specific point of time, however, is challenged to elucidate general dissipation kinetics of pesticides in different plants under variable conditions (Fantke, 2013;

31 Fantke et al., 2014; Jacobsen, 2015). Thus, models have been developed to give deeper insights into the whole system of pesticide-plant-environments (Fantke et al., 2011a). Among them, steady-state and vegetables (Juraske et al., 2011; Trapp et al., 2007), root crops (Fujisawa et al., 2002; Juraske et al., 2009), and fruit trees (Paraíba, 2007). Both types of models require various input parameters to accurately account for kinetics describing pesticide elimination as well as diverse transfer processes. Dynamic models typically rely on solving a set of ordinary first order linear differential equations with constant coefficients as function of time (between pesticide application and crop sampling or harvest), yielding a multi-compartment mass balance problem. Either numerical or analytical methods can be used to solve this problem, or the entire mass balance system can be linearized via model parameterization (Fantke et al., 2012b). As an analytical approach, matrix decomposition (Fantke et al., 2013) provides a convenient and transparent solution.

Using matrix decomposition in a dynamic modelling approach to assess pesticide mass distribution dynamics in different crop-environment systems, the dynamiCROP model was developed as multi-crop 
dynamic plant update model (Fantke and Jolliet, 2016; Fantke et al., 2011a, 2011b). This model has been evaluated against measured residues in wheat (Fantke et al., 2011a; Pang et al., 2016), passion fruit (Juraske et al., 2012), tomato (Fantke et al., 2011a; Feng et al., 2018), potato (Fantke et al., 2011a), rice (Fantke et al., 2011a), apple (Fantke et al., 2011a) and lettuce (Fantke et al., 2011a; Sevigne-Itoiz et al., 2012). The dynamiCROP model describes the whole plant and environment system in a set of interconnected compartments. For example, pesticides distribute, bioaccumulate, translocate, transform, and degrade in soil and air, but also in crop leaf, stem, root and fruit components. This is described by first-order linear differential equations and solved by matrix algebra. Thus, model outputs of pesticide residues represent the plant-environment system at different times after pesticide application.

With the development of economy and education, public food safety incidents draw more and more attention. These incidents, especially pesticide residues in food, threaten consumers' well-being and furthermore undermine the corresponding food market. Such is the anxiety that not only technologies and methods to detect pesticide residues but also dietary risk assessment of pesticides have been called to be implemented worldwide.

There are two methods of assessment, namely, long-term and short-term (or acute) toxicological risk evaluation. Of these, short-term risk evaluation attracts more attention due to the immediate risk at which the short-term pesticide residue intake via food has put the public's health, demanding greater controls on quality of food (Harris et al., 2000). In a long-term evaluation, the estimated daily intake (EDI) of various pesticides is compared with the acceptable daily intake (ADI) issued by World Health Organization (WHO)/ Food and Agriculture Organization of the United Nations (FAO) Joint Meeting on Pesticide Residue (JMPR). Similarly, in short-term evaluations of dietary risk, the estimated shortterm intake (ESTI) of pesticides is compared with acute reference doses (ARfD) to illustrate potential acute health risks for consumers.

Mainly, dietary risk evaluations rely on field trials. However, due to enormous labor, time, solvents and other materials use, field trials cannot provide efficient data for dietary risk assessments for pesticides applied across various crops under different environmental conditions. More importantly, the 
71 low applied quantity of pesticides (usually in the range of $\mathrm{mg} / \mathrm{m}^{2}$ ) following good agricultural practice

72 in field trials (Pang et al., 2016) results in low highest residue levels (HRs) in plants that are difficult to

73 evaluate experimentally. Measured residues are often near or below the analytical detection limits,

74 which greatly contributes to deviation and uncertainty (Pang et al., 2016). Thus, field trials cannot

75 provide sufficient data for dietary risk assessment when comparing multiple pesticides or crops. In order

76 to address this gap, we combined our field trials for three major food crops with the application of the

dynamiCROP model, which could give the maximum HR through modelling simulation across various

pesticide-crop combinations. Related model results can be used as input for screening-level dietary risk evaluation where field trials are difficult to obtain or lacking. However, there has been no such application yet for important staple crops which were treated with the pesticide of thiamethoxam (CAS: 153719-23-4), which is selected in the present study (chemical structure is given in Table 1). Thiamethoxam is a second-generation neonicotinoid insecticide. By binding to nicotinic acetylcholine receptors in the central nervous system, it is presently one of the most effective pesticides for the pest control of such aphids, whiteflies and thrips. It has been recommended for various agricultural crops with global soil, foliar and seed treatments. Its main metabolite is clothianidin (CAS: 210880-92-5) (Table S1) with a maximum occurrence fraction in soil of 35.6\%. Liquid chromatography (LC) (AbdAlrahman, 2014; Jyot and Singh, 2017; Ramasubramanian et al., 2012; Zhou et al., 2006) and LC-MS (Rahman et al., 2015; Valverde et al., 2016) have been widely used to determine residue and dissipation of thiamethoxam (Gupta et al., 2008; Karmakar and Kulshrestha, 2009; Li et al., 2007; Liu et al., 2009; Pareja et al., 2012; Singh and Kulshrestha, 2005). Efforts have also focused on understanding the degradation of thiamethoxam as well as its metabolites (Fan and Shi, 2017; Hilton et al., 2016; Yang et al., 2014). Besides, based on field trials, the dietary risk has been evaluated e.g. in mango (Bhattacherjee and Dikshit, 2016) and tomato (Malhat et al., 2014). However, despite various existing studies focusing on environmental distribution and residue kinetics of thiamethoxam, residue studies for various important food crops are currently still missing, highlighting the need for complementary experimental data and modelling in support of assessing related potential risks for consumers and the environment. 
In the present work, field trials were combined with dynamic modelling to study the environmental

fate and behavior of thiamethoxam in wheat, lettuce and tomato as example crops, among which only

for tomato specific experimental residue studies are currently available in the scientific literature, while missing for the other two crops. Based on HRs from modelling and our own field trials, the acute dietary risk of thiamethoxam was evaluated in the three crops. This is the first dietary risk assessment based on not only on specific experimental data, but also evaluation in complementary computational simulation for this pesticide. This is in line with recommendations to complement and align experimental and modeling results to provide consistent decision support (Fantke et al., 2016).

\section{CAS NO.}

Structure

Molecular weight $(\mathrm{g} / \mathrm{mol})$

Octanol-water partition coefficient

Air-water partition coefficient

$\mathrm{t}_{1 / 2}{ }^{\mathrm{a}} \operatorname{soil}(\mathrm{d})[1]$

$\mathrm{t}_{1 / 2} \mathrm{a}^{\mathrm{w}}$ wheat (d) [2]

$\mathrm{t}_{1 / 2}$ atomato (d) [2]

$\mathrm{t}_{1 / 2}{ }^{\mathrm{a}}$ lettuce (d) [2]

$\mathbf{M}_{\text {applied }}$ in model and experiment $\left(\mathrm{mg} / \mathrm{m}^{2}\right)$

The lowest $\mathrm{MRL}^{\mathrm{b}}\left(\mathrm{mg} \mathrm{kg}^{-1}\right)$
$153719-23-4$

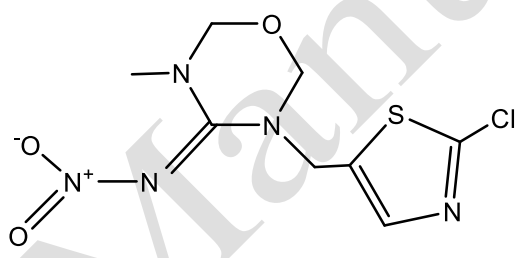

291.71

0.74

$1.9 \times 10^{-13}$

50

3.3

4.5

2.6

0.11 (wheat), 0.11 (lettuce), 1.98 (tomato)

0.02 (wheat, China), 0.2 (tomato, Korea), 3 (lettuce, China)

${ }^{a}$ half-lives used in model

[1]Pesticide Properties Database (https://sitem.herts.ac.uk/aeru/ppdb)

[2] Fantke et al. (2014)

${ }^{\mathrm{b}} \mathrm{MRL}=$ maximum residue limits

Table 1. Physico-chemical properties, degradation half-lives and applied substance mass applied in model for thiamethoxam. 
2.1 Chemicals and solution preparation

Thiamethoxam and its main metabolite clothianidin were purchased from Beijing Qinchengyixin

Co. Ltd. (Beijing, China). Acetonitrile and formic acid (LC grade) were from Fisher Scientific (MA, USA). Anhydrous magnesium sulphate $\left(\mathrm{MgSO}_{4}\right)$, sodium chloride $(\mathrm{NaCl})$, and acetic acid of analytical

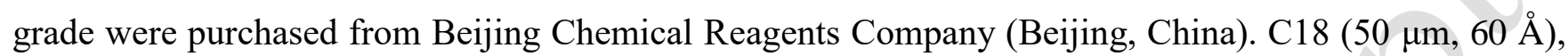
primary secondary amine (PSA) $(40-60 \mu \mathrm{m})$ and graphitized carbon black (GCB) (120-400 mesh) were purchased from Agela Technologies (Tianjin, China). The stock solutions of thiamethoxam and clothianidin $(1 \mathrm{mg} / \mathrm{mL})$ were prepared and kept at $-20^{\circ} \mathrm{C}$ in the dark.

2.2 Field trials

Field trials were conducted according to NY/T 788-2004 (Guideline on pesticide residue trials) (Ministry of Agriculture P.R. China). The field trials were carried out at three sites, namely site A of Beijing $\left(116.46^{\circ} \mathrm{E}, 39.92^{\circ} \mathrm{N}\right)$, site $\mathrm{B}$ of Shandong province $\left(120.42^{\circ} \mathrm{E}, 36.58^{\circ} \mathrm{N}\right)$, and site $\mathrm{C}$ of Anhui province $\left(116.93^{\circ} \mathrm{E}, 34.19^{\circ} \mathrm{N}\right)$ in China. The characteristic properties of soil used in the field, such as $\mathrm{pH}$

values were $6.73,7.32$ and 6.79 for site $\mathrm{A}, \mathrm{B}$ and $\mathrm{C}$, respectively. In addition, organic matter contents of soil were $2.7 \%, 3.9 \%$ and $1.7 \%$ for site $\mathrm{A}, \mathrm{B}$ and $\mathrm{C}$, respectively. The average temperature of three sites

131 during the experimental period was in the range of 20 to $24{ }^{\circ} \mathrm{C}$ in the field trial years of 2016 and 2017. 132 The detailed field trials information were shown in Table S2.

133 For wheat, $0.1 \%$ granule of thiamethoxam was tested to investigate residue dynamics. The 134 recommended dosage was 450-750 grams active ingredient per hectare (g a.i./ha). Residue level trials 135 adopted the high dosage as well as 1.5 folds (1125 $\mathrm{g}$ a.i./ha) with one application before planting. 136 Representative wheat grains at the harvest time $(53 \mathrm{~d})$ were collected.

137 For lettuce, $25 \%$ water dispersion granule of thiamethoxam with recommended dosage of 15-30 g 138 a.i./ha was utilized. The high dosage as well as 1.5 folds (45 g a.i./ha) with two and three times of 
application were adopted. Representative samples with pre-harvest intervals (PHIs) of 3, 5, 7 days after

140 the last application were collected. g a.i./ha was utilized. The high dosage as well as 1.5 folds (94.5 $\mathrm{g}$ a.i./ha) with three and four times application at the peak period of whitening diseases were adopted. Representative samples with PHIs of $1,3,5$ days after the last application were collected.

All samples were stored in dark at $-20^{\circ} \mathrm{C}$ for further analysis.

2.3 Sample preparation by QuEChERS (quick, easy, cheap, effective, rugged and safe)

Homogenized wheat grain $(5 \mathrm{~g})$, lettuce $(5 \mathrm{~g})$ and tomato $(5 \mathrm{~g})$ were extracted by $10 \mathrm{~mL}$

grain and tomato and $2 \mathrm{~mL}$ to lettuce. The salting out step used anhydrous $\mathrm{NaCl}(1 \mathrm{~g})$ and $\mathrm{MgSO}_{4}(4 \mathrm{~g})$

with vortexing for $1 \mathrm{~min}$. After centrifugation at 1, 600 relative centrifugal force (rcf) for $3 \mathrm{~min}, 1.5 \mathrm{~mL}$

of the supernatant was transferred and purified by dispersive solid phase extraction and vortexing for 1

min. Different matrices adopted different combinations of sorbents, i.e. $100 \mathrm{mg}$ PSA, $20 \mathrm{mg}$ GCB and

$150 \mathrm{mg} \mathrm{MgSO}_{4}$ for wheat grain, $100 \mathrm{mg} \mathrm{PSA}, 100 \mathrm{mg} \mathrm{C18,10} \mathrm{mg} \mathrm{GCB}$ and $150 \mathrm{mg} \mathrm{MgSO}_{4}$ for lettuce, and $150 \mathrm{mg}$ PSA, $5 \mathrm{mg}$ multi-walled nanotubes and $150 \mathrm{mg} \mathrm{MgSO}_{4}$ for tomato.

\subsection{LC-MS/MS analysis}

All experiments were performed with Agilent 1260 infinity LC system (Agilent Technologies,

USA) with a Poroshell 120 EC-C18 column (Agilent Technologies) $(50 \mathrm{~mm} \times 3.0 \mathrm{id}, 2.7 \mu \mathrm{m})$. Two

160 analytes were separated with a mobile phase consisting of $0.2 \%$ formic acid and acetonitrile (30:70).

161 The injection volume for all samples was $5 \mu \mathrm{L}$ and the column temperature was $40{ }^{\circ} \mathrm{C}$.

Agilent 6420 triple quadrupole mass spectrometer (Agilent technologies) was operated in positive 163 electrospray ionization multi-reaction monitoring mode (Table S3). Other operating conditions were as 
164 follows: capillary voltage $4000 \mathrm{~V}$, nebulizer pressure 45 psi, drying gas $\left(\mathrm{N}_{2}\right)$ flow rate $11 \mathrm{~L} / \mathrm{min}$, and dry 165 gas temperature $350{ }^{\circ} \mathrm{C}$.

166

2.5 Model application

The dynamic model of dynamiCROP (http://dynamicrop.org) developed by Fantke et al. (2011a) were used in this work. This model was extended to include six crop types covering a large fraction of the worldwide human consumption (Fantke et al., 2011b), and was applied in the present study for wheat, lettuce and tomato to evaluate the evolution, distribution and whereabouts of thiamethoxam between pesticide application and crop harvest. The input data included the selection of pesticide name and crop name as well as the applied amount. The output data included residues in different compartments.

\subsection{Dietary risk assessment}

results as described in equation 1:

$$
\mathrm{EDI}=\frac{\sum S T M R \times \text { Food intake }}{\text { Body weight }}
$$

ESTI is calculated from HR obtained in field trials (or highest theoretical residue levels obtained from

181 dynamic modeling), the daily intake of the highest large portion of food (LP), and unit weight (Ue) 182 following equations 2 :

$$
\mathrm{ESTI}= \begin{cases}\frac{H R \times L P}{B o d y w e i g h t} & \text { if no } U e \\ \frac{U e \times H R \times \vartheta+(L P-U e) \times H R}{B o d y \text { weight }} & \text { if } U e<L P \\ \frac{H R \times L P \times \vartheta}{B o d y \text { weight }} & \text { if } U e>L P\end{cases}
$$

where $\vartheta$ is the variability factor.

\section{Results and Discussion}


The dynamiCROP model consists of source compartments (relevant for pesticide application) and

receiving compartments (relevant for residues in harvest), i.e., air, soil, leaf surface, fruit surface and leaf, fruit, stem, root, respectively. According to the modelled processes, thiamethoxam firstly

191 distributed among four source compartments according to the characteristic of plants and pesticides.

192 Thiamethoxam then transferred, diffused, transformed and degraded in all included compartments. The 193 modelled mass evolution of thiamethoxam in wheat, lettuce and tomato was shown in Figure 1. Mass 194 evolutions included mainly two parts, the initial diffusion and transferring part, and the longer term 195 decreasing trend driven by degradation. After application, thiamethoxam in air, soil, leaf surface and 196 fruit surface decreased due to diffusion and transfer. Thiamethoxam started entering leaf, stem, root, and 197 fruit quickly and then accumulated in these compartments over the first five days. More specifically, 198 after initial distribution, the mass in the crops increased via gas exchange, root uptake, and translocation 199 within the crops. Pesticide mass increased until a maximum was reached, e.g. for fruit, ranging from 16 $200 \mathrm{~g} / \mathrm{kg}$ in tomato to $300 \mathrm{~g} / \mathrm{kg}$ in wheat (see Table 2). From the maximum levels, masses decreased more or 201 less exponentially in all compartments, mainly driven by degradation processes, which was in line with 202 other studies (Jacobsen et al., 2015). It was characteristic that the dynamics in lettuce were driven by 203 soil processes, which was consistent with the longest residence time (40.77 d) in Table 2. The studied 204 metabolite clothianidin generally also showed the initial diffusion and long term decreasing trend that 205 was described for thiamethoxam (Figure S1). In dynamiCROP, any metabolite can be run by first 206 identifying the fraction of this metabolite in relation to the parent compound mass applied and then run 207 this fraction of metabolite as separate chemical in dynamiCROP. 


\begin{tabular}{ccccc}
\hline & & wheat & lettuce & tomato \\
\hline & Air & 0.08 & 0.07 & 0.08 \\
& Soil & 1.21 & 40.77 & 1.06 \\
& Leaf surface & 0.88 & 0.68 & 0.86 \\
residence time & Fruit surface & 4.74 & 3.70 & 2.96 \\
$\mathrm{t}^{\mathrm{b}}(\mathrm{d})$ & Leaf & 3.29 & 3.70 & 6.31 \\
& Fruit & 4.67 & 3.70 & 6.58 \\
& Stem & 0.12 & 0.01 & 0.19 \\
& Root & 0.09 & 0.01 & 0.05 \\
\hline $\begin{array}{c}\text { residue max } \\
(\mathrm{mg} / \mathrm{kg})\end{array}$ & Leaf & $1.0 \times 10^{6}$ & $1.4 \times 10^{5}$ & $1.6 \times 10^{4}$ \\
& Fruit & $3.0 \times 10^{5}$ & & \\
\hline $\begin{array}{c}\text { Harvest fraction } \\
\text { max }\end{array}$ & Stem & $2.6 \times 10^{5}$ & 33 & 0.04 \\
& Leaf & 0.08 & 0.4 & \\
\hline & Fruit & 0.39 & $1.3 \times 10^{-5}$ & \\
\hline & Stem & 0.07 & & \\
\hline
\end{tabular}

$221 \quad{ }^{a} t_{m, \max }=$ maximal mass of pesticides occurring time in plants (d).

$222{ }^{b} t=$ residence time of pesticides in plants (d).

223 Table 2. Modelled parameters in spatial and temporal distribution of thiamethoxam in wheat, lettuce, 224 and tomato. (Data from DynamiCROP) 

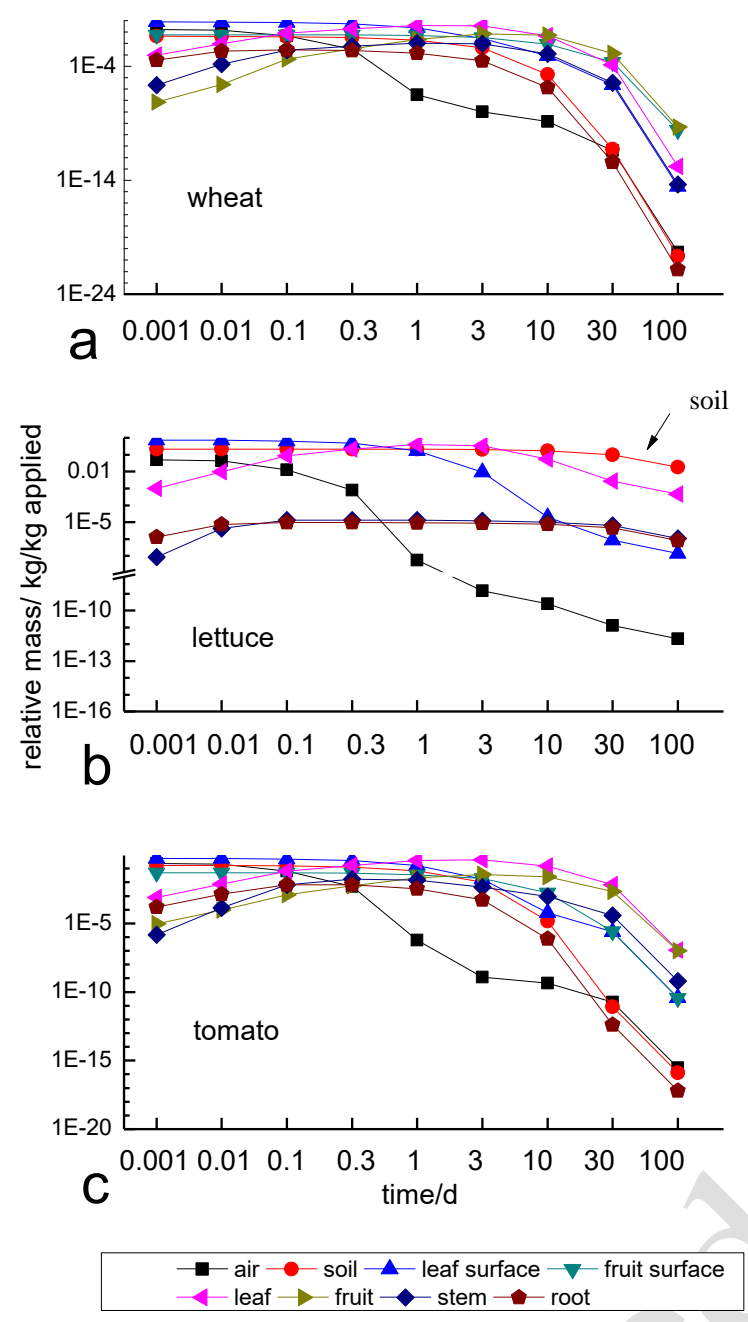

Figure 1. Evolution of masses of thiamethoxam with main driving compartment in wheat (a), soil for lettuce (b), and tomato (c). (Data from DynamiCROP)

2313.2 Modelled contribution of source compartments to crop residue levels

After application, thiamethoxam distributed in four source compartments of air, soil, leaf surface

233 and fruit surface. Herein, for lettuce, it was more convenient to recognize leaves and stem as edible crop 234 parts. In field trials, residues in leaves and the edible crop parts have been paid great attention to 235 considering two important parameters, namely the dissipation half-lives and maximum residue limits 236 (MRLs). Thus, the contribution of the four source compartments on crop residues at different times 237 were quantified by modelling simulation and shown in Figure 2. For residues in leaf, the largest 
contribution came from leaf surface for wheat and tomato, and from soil for lettuce, respectively. This

was mainly related to differences in crop characteristics, where different crops showed different

contributions of pesticide translocation processes (Fantke et al., 2013). The evolution of pesticide

residues in edible fruit was more complex. For wheat, residue in grain mainly came from leaf surface.

For lettuce, residues in leaf mainly came from leaf surface initially and then from soil in the long term.

243 For tomato, different compartments contributed at different points in time, with fruit surface in the

244 initial term, then soil, and finally leaf surface in the longer term. Furthermore, residues in three crops 245 decreased following more or less the same trend over time (Figure 2).

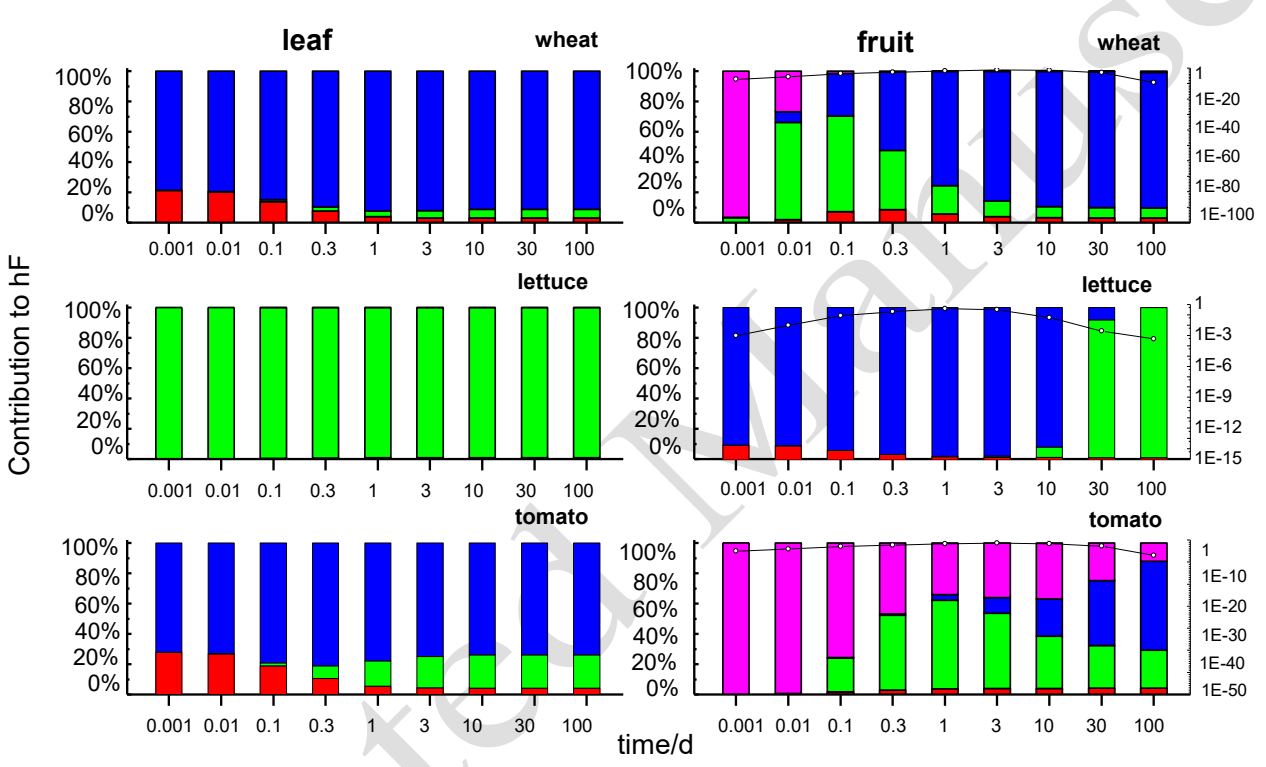

Figure 2. Evolution of contribution of initial thiamethoxam masses in air, soil, leaf surface, and grain surface to masses in leaf and fruit. (Line describing evolution of the harvest fractions in the edible crop parts) (Data from DynamiCROP)

\subsection{Modelled whereabouts of thiamethoxam with soil and foliar application}

Pesticides are usually applied by either foliar spray or soil spray. Different application methods resulted in different distribution, transfer, and degradation dynamics. To illustrate the fate of the pesticide relying on application method, Figure 3 illustrated the whereabouts of thiamethoxam with soil and foliar spray. The mass evolutions of thiamethoxam were different within different crops as well as 
application methods. For soil spray, most thiamethoxam mass went to soil at first, then was transferred

to leaf and fruit gradually in wheat and tomato. In lettuce, almost all the pesticide mass at a specific time

point was retained in the leaf.

The distribution of thiamethoxam with foliar spray was different. Thiamethoxam quickly

transferred from leaf surface to leaf at first. In the longer term $(100 \mathrm{~d})$, thiamethoxam migrated to fruit

for wheat and tomato, and to soil for lettuce. The pesticide fraction in fruit in wheat and lettuce

263 increased and followed a long time decrease, with a maximum of 0.84 at $30 \mathrm{~d}$ in wheat, and 0.97 at $3 \mathrm{~d}$

264 as well as 0.99 at $10 \mathrm{~d}$ for lettuce. At these $3 \mathrm{~d}$ and $10 \mathrm{~d}$ in lettuce, the total fractions of pesticide in leaf

265 surface with foliar spray application were 0.44 and 0.067 , respectively, due to possible dissipation. For

tomato, the pesticide fraction in fruit increased (in leaf) along the time and the ratio at $30 \mathrm{~d}$ was 0.16 .

Hence, whereabouts of thiamethoxam demonstrated different temporal and spatial distribution and

translocation process after application in the different crops.
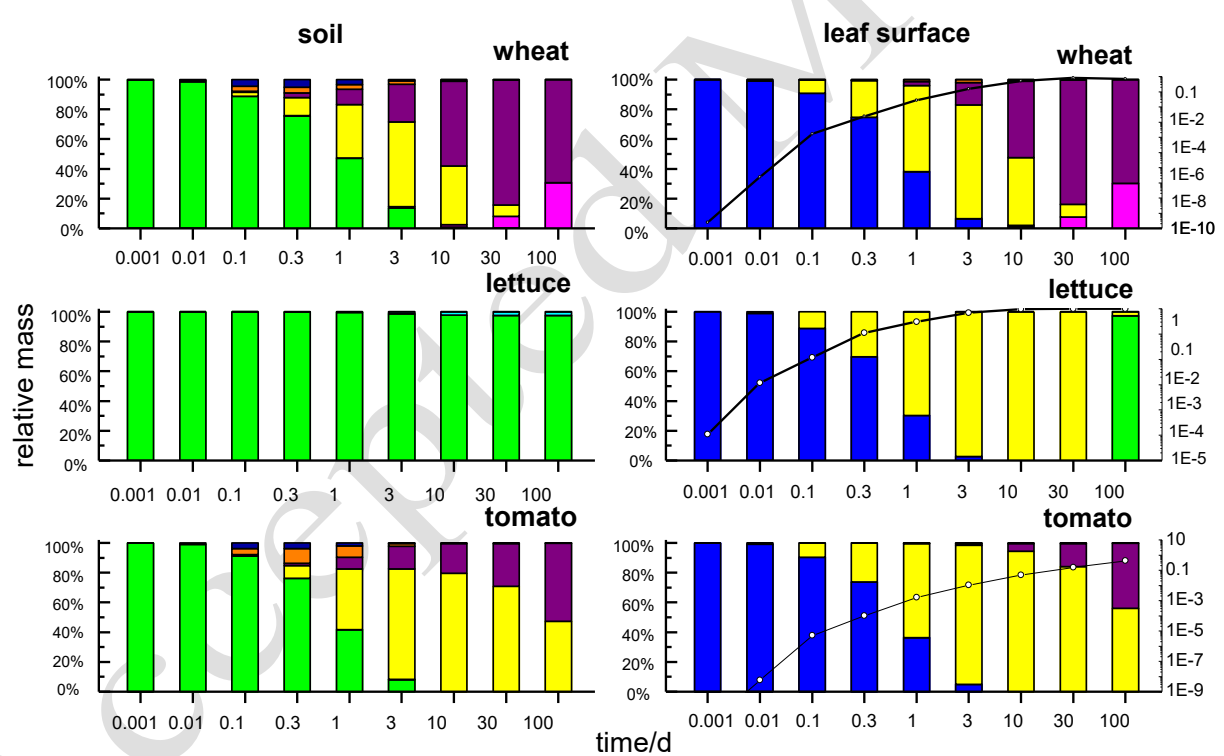

Figure 3. Evolution of whereabouts of applied thiamethoxam ending up as residue in wheat, lettuce and tomato after soil and foliar spray application. (Line describing evolution of fraction in the edible crop parts related to total residues mass for thiamethoxam after foliar spray application) (Data from DynamiCROP) 
Presently, pesticide residue analysis is preferred using QuEChERS LC-MS due to its advantages of satisfactory recoveries, high efficiency, high throughput, and low costs. Hence, QuEChERS LC-MS methods have been established for thiamethoxam as well as its metabolite clothianidin in three matrices. Matrix matched calibration were with acceptable quantitation, satisfactory recoveries and limits of 281 quantitation (LOQs) in wheat grain, lettuce and tomato (Table S4). Thiamethoxam and clothianidin had good linearity in wheat grain, lettuce and tomato in the range of 0.005 to $5 \mu \mathrm{g} / \mathrm{mL}$. Recoveries in three 283 fortified levels in three matrices were in the range of 70 to $110 \%$ (Table S4) with RSD\% in the range of $2843.4 \%-11.3 \%$ in all cases. The reason why the quantitation included its metabolite was due to the 285 definition of residue for thiamethoxam by JMPR, namely, the sum of both two considering the 286 molecular weight ratio of 1.168. Thus, the developed QuEChERS LC-MS and matrix matched 287 calibration could provide reliable residue levels for thiamethoxam. The maximum residues were 288 consistent with model results across studied crops (Table 3).

In field trials, real formulations were applied to different crops with doses as low as 0.11 to 1.98 $\mathrm{mg} / \mathrm{m}^{2}$ (Table 1) following good agricultural practice to imitate real world applications. Representative samples of wheat, lettuce and tomato were analyzed by the developed QuEChERS LC-MS method.

Residue levels in different matrices and with different applications and pre-harvest intervals (PHIs) were obtained (Table S5). For wheat and lettuce, residues were lower than LOQ irrespectively of PHIs.

294 For tomato, HRs were higher than LOQ in the range of 0.19 to $0.43 \mathrm{mg} \mathrm{kg}^{-1}$ with different PHIs. 295 Generally, HRs decreased with the increase of PHIs for all crops.

Residue levels in wheat and lettuce were so low that they could not be detected. Usually, HR was thus maximized to be LOQ for safety concerns. Thus, deviation and uncertainty occurred due to the reliance on the sensitivity of the utilized method. Here, except HR of tomato, HRs of wheat and lettuce were chosen as the LOQ sum of thiamethoxam and its metabolite after molecular weight transformation. 
Acute dietary risk assessment involves the calculation of ARfD\% with HR. Different countries or

303 districts have different average body weight, food intake and dietary structure. Here, we took China as 304 example country with a population of 1.4 billion and one of the biggest consumers of pesticides 305 worldwide [40].

306 Field trials resulted in HRs of thiamethoxam in wheat grain, lettuce and tomato (Table 3 and Table 307 S4), which were either around LOQs or low with considerable deviation. ARfD\% were calculated based 308 on equation 2 assuming no $U e$ for wheat and $U e<L P$ for lettuce and tomato. The corresponding 309 ARfD $\%$ s for different crops were $0.023 \%$ for wheat grain, $0.034 \%$ for lettuce, and $0.5 \%$ for tomato, 310 indicating that at realistic harvest times, all residues fell well below $1 \%$ of the applied pesticide mass in 311 any edible food crop component.

312 Modelling the pesticide residues in dynamiCROP gave maximum residues (Table 2). After the 313 transformation into dosage, theoretical HRs of modelling with experimental application were calculated 314 and shown in Table 3 and in the range of 0.015 to $0.033 \mathrm{mg} \mathrm{kg}^{-1}$. Due to the intrinsic nature of 315 modelling, deviation and uncertainty of residues were greatly lowered.

316 As shown in Table 3, the obtained ESTIs were in the range of $2.5 \times 10^{-4}$ to $3.9 \times 10^{-4} \mathrm{mg} \mathrm{kg}^{-1} \mathrm{bw}$ and 317 the corresponding ARfD\% were in the range of 0.025 to $0.039 \%$. Modelling results were generally 318 consistent with those based on field trials, indicating that thiamethoxam did not provide any 319 unacceptable dietary risk for humans across these three food crops, which was in line with other studies 320 showing that this pesticide showed little dietary pathway related effects on humans compared to other 321 pesticides (Fantke et al., 2012; Karmakar and Kulshrestha, 2009). This simulation gave an example of 322 acute dietary risk assessment in different plants with fast, cost-efficient, and effective processing. 323 Although this study took thiamethoxam in wheat, lettuce, and tomato as an example, it could be applied 324 to many other future applications of such a system for the analysis of other pesticides and their residues 325 and food crops and other plants in support of regulatory and non-regulatory decision-making. 


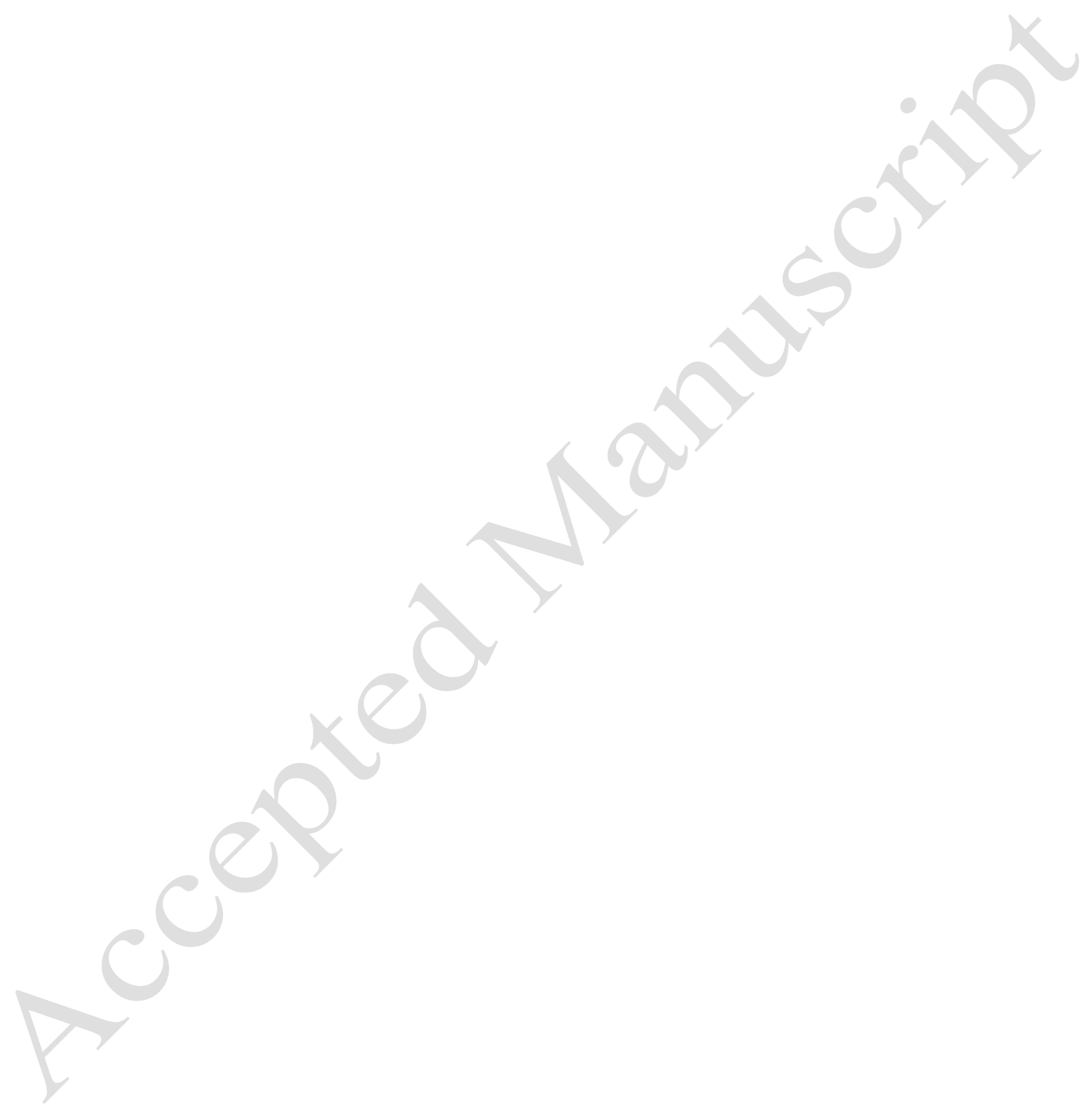




\begin{tabular}{|c|c|c|c|c|c|c|c|c|c|c|}
\hline \multirow[b]{2}{*}{ Crops } & \multirow{2}{*}{$\begin{array}{c}\mathrm{LP}^{\mathrm{a}} \\
\text { (g/person) }\end{array}$} & \multirow{2}{*}{$\begin{array}{l}\mathrm{Ue}^{\mathrm{b}} \\
(\mathrm{g})\end{array}$} & \multirow[b]{2}{*}{$v^{c}$} & \multirow{2}{*}{$\begin{array}{c}\text { Average } \\
\text { body weight } \\
(\mathrm{kg})\end{array}$} & \multirow{2}{*}{$\begin{array}{c}\mathrm{ARfD}^{\mathrm{d}} \\
(\mathrm{mg} / \mathrm{kg} \mathrm{bw})\end{array}$} & \multicolumn{3}{|c|}{ Modelling } & \multicolumn{2}{|r|}{ Field } \\
\hline & & & & & & $\begin{array}{c}\mathrm{HR}^{\mathrm{e}} \\
(\mathrm{mg} / \mathrm{kg})\end{array}$ & $\begin{array}{c}\text { ESTI }^{\mathrm{f}} \\
(\mathrm{mg} / \mathrm{kg} \mathrm{bw})\end{array}$ & ARfD $\%^{\mathrm{g}}$ & $\begin{array}{c}\mathrm{HR}^{\mathrm{e}} \\
(\mathrm{mg} / \mathrm{kg})\end{array}$ & $\begin{array}{c}\text { ESTI }^{\mathrm{f}} \\
(\mathrm{mg} / \mathrm{kg} \mathrm{bw})\end{array}$ \\
\hline Wheat & 732.96 & -- & -- & & & 0.033 & $3.9 \times 10^{-4}$ & 0.039 & 0.02 & $2.3 \times 10^{-4}$ \\
\hline Lettuce & 451.93 & 305.4 & 3 & 63 & 1 & 0.015 & $2.5 \times 10^{-4}$ & 0.025 & 0.02 & $3.4 \times 10^{-4}$ \\
\hline Tomato & 376.77 & 174.6 & 3 & & & 0.032 & $3.6 \times 10^{-4}$ & 0.036 & 0.51 & $5.0 \times 10^{-3}$ \\
\hline
\end{tabular}

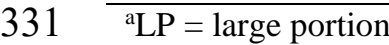

$332{ }^{\mathrm{b}} \mathrm{Ue}=$ unit weight

$333 v_{v}=$ variability factor

$334 \quad{ }^{\mathrm{d}} \mathrm{ARfD}=$ acute reference dose

$335{ }^{\mathrm{e}} \mathrm{HR}=$ the highest residue level

$336 \quad{ }^{\mathrm{f}} \mathrm{ESTI}=$ the estimated short-term intake

$337{ }^{\mathrm{g}} \mathrm{ARfD} \%=\mathrm{ESTI} / \mathrm{ARfD} \times 100$

338 Table 3. Acute dietary risk assessment of thiamethoxam by field trials and computational simulation in China 


\section{Conclusions}

The present study explored the possibility of using a combination of field trials and dynamic modelling, allowing for both fast and reliable data processing as well as direct and transparent 348 demonstration of the evolution, distribution and source apportionment of pesticides in the plant349 environment system. The results provided highest residue levels of thiamethoxam for acute dietary risk 350 assessment in wheat, lettuce and tomato, consistent with the outcome of confirmatory LC-MS. This 351 simulation gave an example of acute dietary risk assessment in different plants with fast, cost-efficient, 352 and effective processing. Although this study took thiamethoxam in wheat, lettuce, and tomato as an 353 example, it can be applied to other applications of such a system for the analysis of other pesticides and 354 their residues and food crops and other plants in support of regulatory and non-regulatory decision355 making.

Abbreviation:

Acute reference dose (ARfD); Estimated short-term intake (ESTI); The highest residue levels (HR); Joint meeting on pesticide residue (JMPR); Large portion of food (LP); Liquid chromatography (LC); Mass spectrometry (MS); Supervised trials median residue levels (STMR); Unit weight (Ue);

Variability factor (v)

\section{Acknowledgements}

365 This research was supported by the China Scholarship Council and by the OLCA-Pest project 366 financially supported by ADEME (grant agreement no. 17-03-C0025).

\section{Supporting information description}

369 There are 5 Tables, 1 Figure, 7 pages in total in the supporting material. 
372 Competing financial interest

373 The authors declare no competing financial interest.

374

375

376

377

378

379

380

381

382

383

384

385

386

387

388

389

390

391

392

393

394

395

396 
Figure 1. Evolution of masses of thiamethoxam with main driving compartment in wheat (a), soil for 399 lettuce (b), and tomato (c). (Data from DynamiCROP)

400 Figure 2. Evolution of contribution of initial thiamethoxam masses in air, soil, leaf surface, and grain 401 surface to masses in leaf and fruit. (Line describing evolution of the harvest fractions in the edible crop 402 parts) (Data from DynamiCROP)

403 Figure 3. Evolution of whereabouts of applied thiamethoxam ending up as residue in wheat, lettuce and 404 405 tomato after soil and foliar spray application. (Line describing evolution of fraction in the edible crop parts related to total residues mass for thiamethoxam after foliar spray application) (Data from DynamiCROP)

\section{References}

Abd-Alrahman, S. H. (2014). Residue and dissipation kinetics of thiamethoxam in a vegetable-field ecosystem using QuEChERS methodology combined with HPLC-DAD. Food Chem, 159, 1-4. https://doi.org/10.1016/j.foodchem.2014.02.124

Bhattacherjee, A. K., \& Dikshit, A. (2016). Dissipation kinetics and risk assessment of thiamethoxam and dimethoate in mango. Environ Monit Assess, 188, 165. https://doi.org/10.1007/s10661-016$5160-3$

Fan, Y. J., \& Shi, X. Y. (2017). Characterization of the metabolic transformation of thiamethoxam to clothianidin in Helicoverpaarmigera, larvae by SPE combined UPLC-MS/MS and its relationship with the toxicity of thiamethoxam to helicoverpa armigera larvae. J Chromatogr B, 1061, 349-355. https://doi.org/10.1016/j.jchromb.2017.07.054

Fantke, P., Arnot, J. A., \& Doucette, W. J. (2016). Improving plant bioaccumulation science through consistent reporting of experimental data. J Environ Manage, 181, 374-384. http://doi.org/10.1016/j.jenvman.2016.06.065

Fantke, P., Charles, R., de Alencastro, L. F., Friedrich, R., \& Jolliet, O. (2011). Plant uptake of pesticides and human health: dynamic modeling of residues in wheat and ingestion intake. Chemosphere, 85, 1639-1647. https://doi.org/10.1016/j.chemosphere.2011.08.030 
Fantke, P., Friedrich, R., \& Jolliet, O. (2012). Health impact and damage cost assessment of pesticides in Europe. Environ Int, 49, 9-17. http://doi.org/10.1016/j.envint.2012.08.001

Fantke, P., Gillespie, B. W., Juraske, R., \& Jolliet, O. (2014). Estimating half-lives for pesticide dissipation from plants. Environ Sci Technol, 48, 8588-8602. http://doi.org/10.1021/es500434p

Fantke, P., \& Jolliet, O. (2016). Life cycle human health impacts of 875 pesticides. Int J Life Cycle Assess, 21, 722-733. http://doi.org/10.1007/s11367-015-0910-y

Fantke, P., \& Juraske, R. (2013). Variability of pesticide dissipation half-lives in plants. Environ Sci Technol, 47, 3548-3562. https://doi.org/10.1021/es303525x

Fantke, P., Juraske, R., Antón, A., Friedrich, R., \& Jolliet, O. (2011). Dynamic multicrop model to characterize impacts of pesticides in food. Environ Sci Technol, 45, 8842-8849. http://doi.org/10.1021/es201989d

Fantke, P., Wieland, P., Juraske, R., Shaddick, G., Sevigne-Itoiz, E., Friedrich, R. \& Jolliet O. (2012). Parameterization models for pesticide exposure via crop consumption. Environ Sci Technol, 46, 12864-12872. https://doi.org/10.1021/es301509u

Fantke, P., Wieland, P., Wannaz, C., Friedrich, R., \& Jolliet, O. (2013). Dynamics of pesticide uptake into plants: From system functioning to parsimonious modeling. Environ Modell Softw, 40, 316324. http://doi.org/10.1016/j.envsoft.2012.09.016

FAOSTAT http://www.fao.org/faostat/en/

Feng, X. X., Wang, K., Pan, L. X., Xu, T. H., Zhang, H. Y., \& Fantke, P. (2018). Measured and modeled residue dynamics of famoxadone and oxathiapiprolin in tomato fields. J Agric Food Chem, 66, 8489-8495. http://doi.org/10.1021/acs.jafc.8b02056

Fujisawa, T., Ichise, K., Fukushima, M., Katagi, T., \& Takimoto, Y. (2002). Improved uptake models of nonionized pesticides to foliage and seed of crops. J Agric Food Chem, 50, 532-537. https://doi.org/10.1021/jf010985j

Gupta, S., Gajbhiye, V.T. \& Gupta, R.K. (2008). Soil dissipation and leaching behavior of a neonicotinoid insecticide thiamethoxam. Bull Environ Contam Toxicol, 80, 431-437. http:// doi.org/10.1007/s00128-008-9420-y 
Harris, C. A., Mascall, J. R., Warren, S. F. P., \& Crossley, S. J. (2000). Summary report of the

455

456

457

458

459

460

461

462

463

464

465

466

467

468

469

470

471

472

473

474

475

476

International Conference on pesticide residues variability and acute dietary risk assessment. Food Addit Contam 17, 481-485. http://doi.org/10.1080/026520300412357

Hajslova, J., Cajka, T., \&Vaclavik, L. (2011). Challenging applications offered by direct analysis in real time (DART) in food-quality and safety analysis. Trac-Trend Anal Chem, 30, 204-218. https://doi.org/10.1016/j.trac.2010.11.001

Hilton, M. J., Jarvis, T. D. \& Ricketts, D. C. (2016). The degradation rate of thiamethoxam in European field studies. Pest Manag Sci, 72, 388-397. http://doi.org/10.1002/ps.4024

Jacobsen, R. E., Fantke, P., \& Trapp, S. (2015). Analysing half-lives for pesticide dissipation in plants. SAR QSAR Environ Res, 26, 325-342. http://doi.org/10.1080/1062936X.2015.1034772

Juraske, R., Castells, F., Vijay, A., Muñoz, P., \& Antón, A. (2009). Uptake and persistence of pesticides in plants: measurements and model estimates for imidacloprid after foliar and soil application. $\mathbf{J}$ Hazard Mater, 165, 683-689. https://doi.org/10.1016/j.jhazmat.2008.10.043

Juraske, R., Fantke, P., Ramírez, A. C. R., \& González, A. (2012). Pesticide residue dynamics in passion fruits: Comparing field trial and modeling results. Chemosphere, 89, 850-855. http://doi.org/10.1016/j.chemosphere.2012.05.007

Juraske, R., Vivas, C. S. M., Velásquez, A. E., Santos, G. G., Moreno, M. B. B., Gomez, J. D., Binder, C. R., Hellweg, S. \& Dollos, J. A. G. (2011). Pesticide uptake in potatoes: model and field experiments. Environ Sci Technol, 45, 651-657. https://doi.org/10.1021/es102907v

Jyot, G., \& Singh, B. (2017). Development and validation of an HPLC method for determination of thiamethoxam and its metabolites in cotton leaves and soil. J AOAC Int, 100, 796-803. https://doi.org/10.5740/jaoacint.16-0206

Karmakar, R. \& Kulshrestha, G. (2009). Persistence, metabolism and safety evaluation of thiamethoxam in tomato crop. Pest Manag Sci, 65, 931-937. http:// doi.org/10.1002/ps.1776

Li, M. I., Song, S. E., Jian, J., \& Zhang, X.A. (2007). Dynamics and terminal residue of thiamethoxam on tomato (Lycopersiconesculentum Mill.). Agrochemicals, 46, 477-478. http://en.cnki.com.cn/Article_en/CJFDTOTAL-NYZZ200707016.htm 
Liu, B., Guo, D. L., Mao, J. S., Zhao, S. C., \& Wang, Y. T. (2009). Residue detection and degradation of thiamethoxam in spinach. Agrochemicals, 48, 667-668,674. http://en.cnki.com.cn/Article_en/CJFDTOTAL-NYZZ200909018.htm

484

485

486

Malhat, F. M., Watanabe, H., Loutfy, N. M., \& Ahmed, M. T. (2014). Hazard assessment of the neonicotinoid insecticide thiamethoxam residues in tomato: a prelude to risk assessment profile. Toxicol Environ Chem, 96, 318-327. https://doi.org/10.1080/02772248.2014.932522

Pang, N. N., Cui, Y., \& Hu, J. Y. (2016). Weather dependent dynamics of the herbicides florasulam, carfentrazone-ethyl, fluroxypyr-meptyl and fluroxypyr in wheat fields through field studies and computationalsimulation. Chemosphere,

165 , 320-328. https://doi.org/10.1016/j.chemosphere.2016.09.026

Paraíba, L. C. (2007). Pesticide bioconcentration modelling for fruit trees. Chemosphere, 66, 1468-1475. https://doi.org/10.1016/j.chemosphere.2006.09.017

Pareja, L., Colazzo, M., Pérez-Parada, A., Besil, N., Heinzen, H., Böcking, B., Cesio, V., \& FernándezAlba, A. R. (2012). Occurrence and distribution study of residues from pesticides applied under controlled conditions in the field during rice processing. J Agric Food Chem, 60, 4440-4448. http:// doi.org/10.1021/jf205293j

Rahman, M. M., Farha, W., Abd El-Aty, A. M., Kabir, M. H., Im, S. J., Jung, D. I., Choi, J. H., Kim, S. W., Son, Y. W., Kwon, C. H., Shin, H.C. \& Shim, J. H. (2015). Dynamic behaviour and residual pattern of thiamethoxam and its metabolite clothianidin in Swiss chard using liquid chromatography-tandem mass spectrometry. Food Chem, 174, 248-255. https://doi.org/10.1016/j.foodchem.2014.11.052

Ramasubramanian, T., Paramasivam, M., \& Jayanthi, R. (2012). Rapid and sensitive analytical method for simultaneous determination of imidacloprid and thiamethoxam residues in soils of sugarcane ecosystem by reversed-phase HPLC. Water Air Soil Poll, 223, 6045-6050. https://doi.org/10.1007/s11270-012-1338-7

Rein, A., Legind, C. N., \& Trapp, S. (2011). New concepts for dynamic plant uptake models. Sar QSAR Environ Res, 22, 191-215. https://doi.org/10.1080/1062936X.2010.548829 
508 Sevigne-Itoiz, E., Fantke, P., Juraske, R., Kounina, A., \& Antón-Vallejo, A. (2012). Deposition and residues of azoxystrobin and imidacloprid on greenhouse lettuce with implications for human consumption. Chemosphere, 89, 1034-1041. http://doi.org/10.1016/j.chemosphere.2012.05.066

511 Singh, S. B. \& Kulshrestha, G. (2005). Residues of thiamethoxam and acetamaprid, two neonicotinoid 512 insecticides, in/on okra fruits (Abelmoschusesculentus L). Bull Environ Contam Toxicol, 75, 945513 951. http://doi.org/10.1007/s00128-005-0841-6

514 Trapp, S., Cammarano, A., Capri, E., Reichenberg, F., \& Mayer, P. (2007). Diffusion of PAH in potato 515 and carrot slices and application for a potato model. Environ Sci Technol, 41, 3103-3108. $516 \quad$ https://doi.org/10.1021/es062418o

517 Valverde, S., Ares, A. M., Bernal, J. L., Nozal, M. J., \& Bernal, J. (2016). Simultaneous determination 518 of thiamethoxam, clothianidin, and metazachlor residues in soil by ultrahigh performance liquid 519 chromatography coupled to quadrupole time-of-flight mass spectrometry. J Sep Sci, 40, 1083-1090. 520 https://doi.org/ 10.1002/jssc.201601143

521 Yang, H., Liu, H. J., Hu, Z. B., Liang, J. W., Pang, H. L., \& Yi, B. (2014). Consideration on degradation 522 kinetics and mechanism of thiamethoxam by reactive oxidative species (ROSs) during 523 photocatalytic process. Chem Eng J, 245, 24-33. https://doi.org/10.1016/j.cej.2014.02.016

524 Zhou, Q. X., Ding, Y. J., \& Xiao, J. P. (2006). Sensitive determination of thiamethoxam, imidacloprid 525 and acetamiprid in environmental water samples with solid-phase extraction packed with multi526 walled carbon nanotubes prior to high-performance liquid chromatography. Anal Bioanal Chem, 527 385, 1520-1525. https://doi.org/10.1007/s00216-006-0554-7 


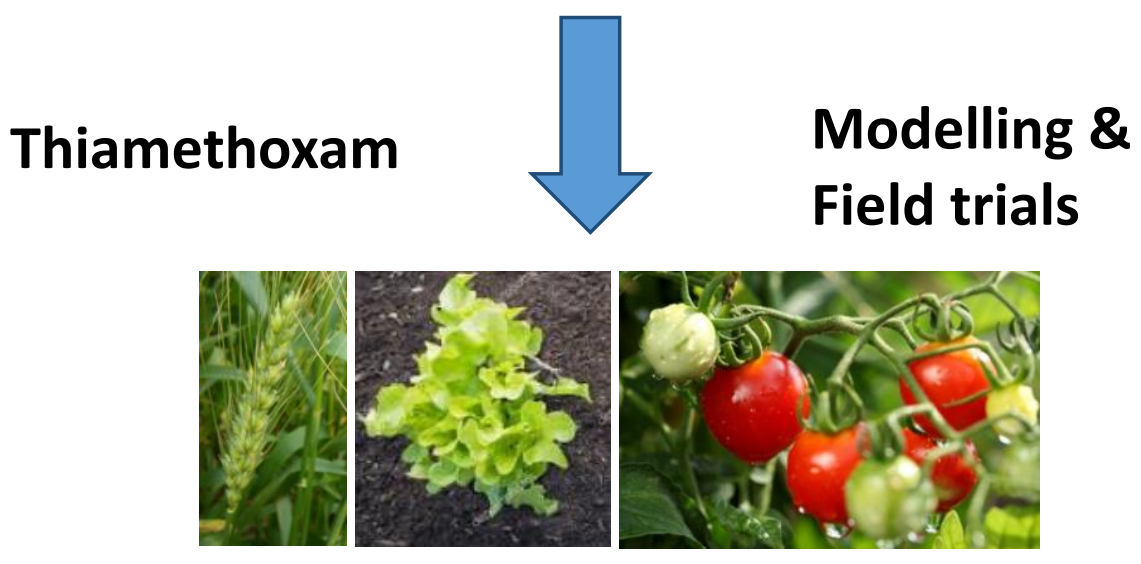

530

Dietary risk assessment

531 Table of contents 